\title{
Estado de las poblaciones del mangle blanco Laguncularia racemosa var. glabriflora (Combretaceae) en el Golfo de Guayaquil, Ecuador
}

\author{
Population status of the white mangrove Laguncularia racemosa var. \\ glabriflora (Combretaceae) in the Gulf of Guayaquil, Ecuador
}

\section{Huber Ricardo Moreira Estrella}

\section{Consultor Independiente}

Recibido de agosto 2020; recibido en forma revisada 26 de agosto 2020, aceptado 3 de octubre 2020 Disponible en línea 20 de diciembre 2020

\begin{abstract}
Resumen
Se realizó un censo de las poblaciones de Laguncularia racemosa var. glabriflora para evaluar su estado en el Golfo de Guayaquil. Durante diciembre 2020 y enero 2021 se establecieron 10 parcelas de forma rectangular, cada una con una superficie de $50 \times 20 \mathrm{~m}\left(1.000 \mathrm{~m}^{2}\right)$, abarcando un área total de 1 hectárea $\left(10.000 \mathrm{~m}^{2}\right)$ en las provincias del Guayas y El Oro, en la costa de Ecuador. Laguncularia racemosa var. glabriflora habita predominantemente manglares ribereños y suelos lodosos (mayoritariamente limo) de textura floja, con salinidad intersticial desde 5 hasta 38 UPS, la mayor densidad poblacional se registró en las localidades que poseen entre 20 a 35 UPS. Las poblaciones registradas poseen poco desarrollo estructural, correspondiendo un $56 \%$ en la categoría diamétrica latizales $(5-9,9 \mathrm{~cm}$ DAP). Las dos variedades de $L$. racemosa son marcadamente simpátricas en el Golfo de Guayaquil. En el $80 \%$ de las parcelas censadas ambas variedades florecen y fructifican simultáneamente, sin embargo, evidencia de variabilidad morfológica o hibridación producto de ambas no ha sido encontrada en campo ni en herbario. Estos resultados sugieren que no habría compatibilidad biológica entre ambas variedades, consecuentemente, se recomienda una revisión de su estatus taxonómico con base en estudios moleculares.
\end{abstract}

Palabras claves: Golfo de Guayaquil, Laguncularia racemosa var. glabriflora, manglares, simpátrica, variedad.

\begin{abstract}
A census of Laguncularia racemosa var. glabriflora was carried out in the Gulf of Guayaquil to assess the status of the populations. During December 2020 and January 2021, 10 rectangularshaped plots were established, each with an area of $50 \times 20 \mathrm{~m}\left(1,000 \mathrm{~m}^{2}\right)$, covering a total area of 1 hectarea $\left(10,000 \mathrm{~m}^{2}\right)$ in the provinces of Guayas and El Oro, in the coast of Ecuador. Laguncularia racemosa var. glabriflora predominantly inhabits riparian mangroves and muddy (mostly silt) soils with a loose texture, and interstitial salinity from 5 to 38 UPS, the highest population density was recorded in localities that have between 20 to 35 UPS. The recorded populations have a low structural development, corresponding to $56 \%$ in the latizal diameter category $(5-9.9 \mathrm{~cm} \mathrm{DBH})$. The two varieties of $L$. racemosa are markedly sympatric in the Gulf of Guayaquil. In $80 \%$ of the surveyed plots they bloom and fructify simultaneously, however, evidence of morphological variability or hybridization among both varieties has not been found in the field neither in herbarium. These results suggest that biological compatibility would not be achieved among both varieties and that consequently, a review of its taxonomic status based on molecular studies is recommended.

Keywords: Guayaquil Gulf, Laguncularia racemosa var. glabriflora, mangroves, sympatric, variety.
\end{abstract}

\section{Introducción}

Los manglares son ecosistemas productivos conocidos por los bienes y servicios ambientales que ofrecen, son sitios de reproducción y refugio de docenas de especies de aves, mamíferos, reptiles, peces, crustáceos, moluscos, insectos y arácnidos y desempeñan el papel de barrera de

* Correspondencia del autor:

E-mail: huber.moreirae@ug.edu.ec amortiguamiento contra el oleaje y vientos marinos. Son sitios donde se produce la filtración del agua y retienen contaminantes, entre ellos a los metales pesados, atenuando su dispersión hacia los océanos (Erazo, 2014), y contribuyen a mantener la calidad de la vida marina y costera. Su vegetación es predominantemente leñosa y posee tolerancia a la salinidad (Murrieta, 2018). 
En tiempos prehispánicos, el aprovechamiento de los manglares se realizaba de manera sustentable por grupos indígenas en la costa de Ecuador y noroccidente de Perú. A la llegada de los españoles, las virtudes de las maderas de los mangles fueron rápidamente notadas por su dureza y durabilidad, la explotación de los mangles en el Golfo de Guayaquil aumentó de forma exponencial, siendo materia prima útil para la construcción de barcos e infraestructura local, la madera de los mangles fue exportada en grandes cantidades desde Guayaquil hacia varias ciudades de Perú (Madsen et al., 2001). Se estima que a nivel mundial la pérdida de bosques de manglar en el siglo pasado fue entre un 30 a $50 \%$, principalmente por actividades de desarrollo urbano y de la acuacultura (Benítez et al., 2018). En Ecuador, los manglares abarcan una extensión aproximada de 157.094,28 ha, se estima que la pérdida de su superficie hasta el 2006 fue de 56.395,9 ha., lo que equivale el $27,7 \%$ de la superficie original (Bravo, 2010).

En Ecuador la formación vegetal de estos ecosistemas está caracterizada por los denominados mangles mayores, los que son: Rhizophora mangle, $R$. racemosa, $R$. $X$ harrisonii (Rhizophoraceae), Hilarianthus germinans (Acanthaceae) y Laguncularia racemosa (Combretaceae); esta última especie posee dos variedades, $L$. racemosa var. racemosa y $L$. racemosa var. glabriflora (Cornejo, 2014).

Laguncularia racemosa var. racemosa se distribuye desde el norte de México hasta la costa noroccidental del Perú en el Pacífico oriental. Este mangle se caracteriza por ser un árbol perennifolio con neumatóforos cortos; sus hojas son simples, opuestas y glabras; posee un par de glándulas en el ápice del peciolo; la inflorescencia es una panícula terminal con pequeñas flores blancas, los cálices son densamente pubescentes; los frutos son drupáceos, longitudinalmente sulcados, densamente pubescentes, de color verde opaco, las semillas poseen embriones fotosintéticos (Cumaná et al., 2010). La variedad glabriflora se caracteriza por tener: un tallo de corteza gris, lisa, lenticelada; las inflorescencias, brácteas florales, cálices y frutos son completamente glabros de color verde brillante y más o menos lustroso. Según las colecciones de herbario esta variedad habita en ambas costas de América tropical, en la costa del Pacífico se encuentra restringida al Golfo de Guayaquil, y se extiende desde la provincia del Guayas en Ecuador, hasta Tumbes en Perú, es un componente que caracteriza a la subunidad florístrica conocida como manglares del Bosque seco deciduo del Pacífico Ecuatorial (Cornejo, 2014). En esta área, L. racemosa var. glabriflora ha estado bajo intensa presión por transformación de hábitat para el establecimiento de piscinas camaroneras por el desarrollo urbano anómalo, este taxón ha sido categorizado según los criterios UICN como vulnerable (VU B2abiii) (Cornejo, 2014).

Laguncularia racemosa var. glabriflora fue colectada por primera vez en la ciudad de Guayaquil en Octubre de 1790 por el naturalista checo Taddaeus Haenke, mientras exploraba el río Daule, como miembro de la expedición de Alejandro Malaspina alrededor del mundo. Inicialmente fue descrita por C. Presl en 1831 (1825-1835) como una nueva especie de mangle con el nombre de Laguncularia glabriflora, y ha permanecido por más de 100 años en la sinonimia bajo L. racemosa hasta la publicación del tratamiento taxonómico de la familia Combretaceae en Flora of Ecuador (Stace, 2007), en el que se ha reestablecido el uso de este taxón pero a nivel de variedad, como Laguncularia racemosa var. glabriflora (op. cit.). La falta de su reconocimiento se atribuye a la ausencia de estudios taxonómicos en las plantas de manglares en Ecuador (Cornejo, 2015).

Laguncularia racemosa var. glabriflora ha sido registrada en: Estero Salado, Estero Mongón y la isla Puná (Cornejo, 2014). En el Estero Salado se han realizado estudios sobre la concentración de metales pesados, entre los que destaca el cadmio (Cd) con una concentración de 0,5 a $12 \mathrm{mg} / \mathrm{Kg}$. Este metal pesado es perjudicial para la sobrevivencia de esta variedad, reportándose que concentraciones de $\mathrm{Cd}(0,5 \mathrm{mg} / \mathrm{L})$, el máximo permitido por la Legislación Ecuatoriana para suelo (Texto unificado de la legislación ambiental, acuerdo ministerial 097A), inhibe el crecimiento radicular y dificulta la sobrevivencia de $L$. racemosa var. glabriflora (Benavidez et al., 2018).

Estudios adicionales del mangle blanco se han realizado a nivel nacional, pero sin especificar la variedad a las que pertenecen las poblaciones, identificando simplemente a Laguncularia racemosa, por lo que existe un vacío enorme sobre el estado de las poblaciones de Laguncularia racemosa var. glabriflora. El presente trabajo tiene como objetivo evaluar el estado actual de Laguncularia racemosa var. glabriflora, con el propósito de generar la información básica y contribuir a su manejo y a la conservación de la diversidad florística de los manglares y del Ecuador.

\section{Materiales y Métodos}

\section{Área de estudio}

Las localidades muestreadas se encuentran distribuidas a lo largo de las provincias Guayas y El Oro, están ubicadas desde $81^{\circ} 00^{\prime}$ 'W hasta $79^{\circ} 42^{\prime} \mathrm{W}$ y desde $2^{\circ} 19^{\prime}$ $S$ hasta $3^{\circ} 29^{\prime} \mathrm{S}$; se encuentran influenciadas por las mareas, durante el día tienen dos bajamares y dos pleamares (INOCAR, 2021; Fig. 1).

\section{Metodología}

Se realizaron las siguientes actividades: i) Compilación bibliográfica de literatura publicada como artículos, revisiones taxonómicas o libros, donde se trata a Laguncularia racemosa var. glabriflora. ii) Revisión de las colecciones del herbario GUAY de la Universidad de Guayaquil, en esta institución existe la mayor concentración de especímenes de la variedad en estudio obtenidas en la costa del Pacífico este a nivel 


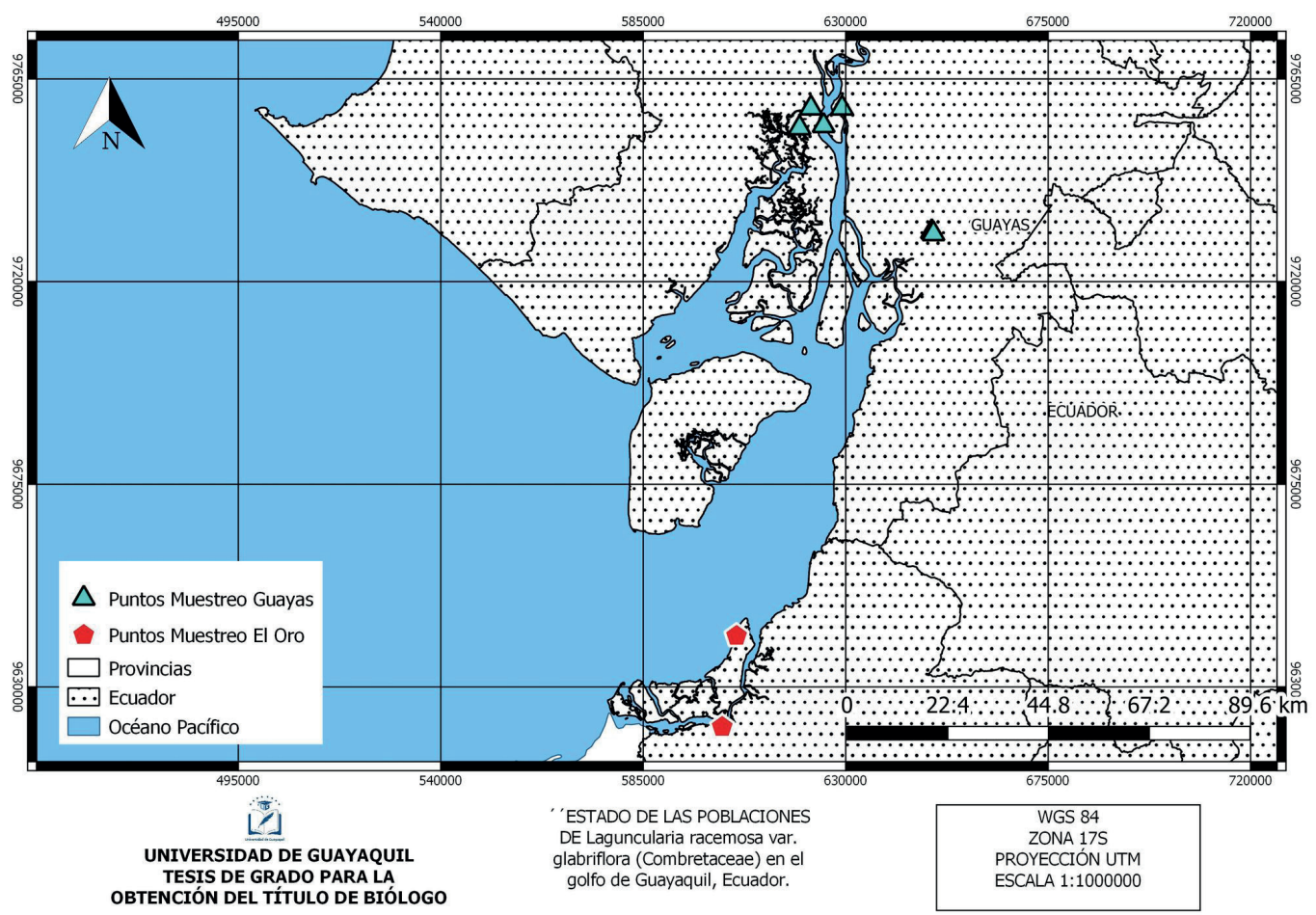

Figura 1. Sitios de muestreos de las poblaciones de Laguncularia racemosa var. glabriflora en el Golfo de Guayaquil

nacional e internacional (Cornejo, 2014). iii) Con base en la revisión bibliográfica y de las colecciones de herbario, se procedió a la comprobación en campo de la presencia y localización de las poblaciones de la variedad en estudio.

\section{Fase de campo}

Localizar las poblaciones de Laguncularia racemosa var. glabriflora en el Golfo de Guayaquil.

La fase de campo se realizó durante diciembre 2020 y enero 2021, estos meses corresponden al final de la estación seca e inicio de la estación lluviosa, respectivamente. Se establecieron 10 parcelas de forma rectangular, cada una con una superficie de 50

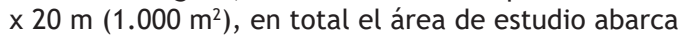
1 hectárea $\left(10.000 \mathrm{~m}^{2}\right)$ en el Golfo de Guayaquil. Ocho parcelas se establecieron en la provincia del Guayas y dos en la provincia de El Oro. En campo, con el uso de un GPS marca Garmin se registró la posición geográfica en el extremo izquierdo de cada parcela.

Una vez establecidas las parcelas se procedió a levantar los datos en campo de las poblaciones presentes de la variedad estudiada. Para el censo poblacional de $L$. racemosa var. glabriflora, se tomaron en cuenta los individuos con un diámetro a la altura del pecho (DAP) mayor a $2,5 \mathrm{~cm}$ y exclusivamente que tuvieran la presencia de flores y frutos, debido a que éstas partes reproductivas son las únicas que presentan los caracteres diagnósticos para el reconocimiento del taxón en estudio y permiten obtener un $100 \%$ de confianza en la identidad de los individuos registrados.

A cada individuo censado al interior de las parcelas se le tomó los siguientes datos:

- $\operatorname{DAP}(\mathrm{cm})$, esto es, a 1,3 m de alto,

- Alto total, esto es desde la base del tallo hasta el ápice de la copa,

- Diámetro de copa $\left(\mathrm{m}^{2}\right)$.

Registrar del patrón de distribución de Laguncularia racemosa var. glabriflora en el Golfo de Guayaquil. El patrón de distribución de Laguncularia racemosa var. glabriflora se determinó aplicando en campo la metodología que se detalla a continuación.

Para establecer el tipo fisiográfico de los manglares se siguió la clasificación de Lugo y Snedeaker (1974) y modificado por Cintrón (1980), esta considera los siguientes tipos de manglar: (i) de borde, (ii) ribereño, y (iii) de cuenca. El grado de desarrollo estructural del manglar en que habita la variedad estudiada se determinó conforme a la metodología empleada según Dinaf-Clirsen (1991), esta considera a la altura de la formación vegetal, clasificándola en 3 categorías: (i) bajo (hasta $5 \mathrm{~m}$ ), (ii) medio (5 a 20 m), y (iii) alto (20 a $40 \mathrm{~m}$ ). 
Para los sustratos se revisó en campo la textura del suelo, consistencia y si hay elementos introducidos o modificaciones estructurales que los habrían alterado.

Para determinar la textura del suelo se usó el método manual descrito por Garrido (1993), este consiste en tomar una muestra de suelo y formar una figura cilíndrica que según su grosor y forma se puede definir la textura del suelo. Según lo observado se clasificó a los suelos en: (i) lodoso (mayoritariamente limo), (ii) arenoso (mayoritariamente arena), (iii) rocoso (mayoritariamente graba y rocas) y (iv) sustratos artificiales (islas flotantes). Se tomó datos de salinidad intersticial en los sustratos de cada parcela, para este propósito se utilizó el método aplicado por Idrogo (2016), con algunas modificaciones en el uso del equipo. Se usó un tubo de plástico de 30 $\mathrm{cm}$ de largo con $22 \mathrm{~cm}$ de circunferencia, el cual se introdujo en el suelo en marea baja, luego se lo retiró con el sedimento dejando un hoyo de unos $30 \mathrm{~cm}$ de profundidad, se esperó que se filtrara el agua en el hoyo y luego con una jeringa se tomó la muestra de agua, conservándola en frío, para después ser analizada con un refractómetro, determinando el valor de la salinidad intersticial en UPS.

\section{Análisis de datos.}

Determinación el estado de las poblaciones de Laguncularia racemosa var. glabriflora en el Golfo de Guayaquil.

Los individuos censados fueron clasificados en tres categorías diamétricas según Cintrón y Schaeffer (1992), como se detalla a continuación (Tabla 1).

En las 10 parcelas establecidas en el Golfo de Guayaquil, se observó el estado fenológico de los frutos, es decir si eran inmaduros o maduros. Además, en cada parcela se verificó la presencia de los individuos de $L$. racemosa var. racemosa, determinando la frecuencia en donde las dos variedades de $L$. racemosa se encontraban presentes. Luego se revisó en campo si los individuos censados tenían características intermedias como un indicador de variabilidad de los caracteres morfológicos o como evidencia de hibridación de las dos variedades de mangle blanco.

\section{Densidad (D)}

La densidad se entiende como el número de individuos en un área determinada, esta se expresa en individuos

Tabla 1. Categorías diamétricas de las poblaciones de Laguncularia racemosa var. glabriflora en el Golfo de Guayaquil.

\begin{tabular}{cc}
\hline $\begin{array}{c}\text { Categoría } \\
\text { poblacional }\end{array}$ & $\begin{array}{c}\text { Categoría diamétrica (DAP } \\
\mathrm{cm})\end{array}$ \\
\hline Brinzales & $2,5-4,99$ \\
Latizales & $5-9,9$ \\
Fustales & $\geq 10$ \\
\hline
\end{tabular}

por hectárea (individuos $/ \mathrm{ha}^{-1}$ ), aplicando la siguiente fórmula (Matteucci y Colma, 1982).

\section{$\mathbf{D}=\mathbf{N} / \mathbf{A}$}

Donde:

$\mathrm{N}=\quad$ Número de individuos presentes en la parcela

$A=\quad$ Extensión de área de muestreo

\section{Densidad Relativa $\left(D_{R}\right)$}

La densidad relativa se utiliza para comparar la densidad de una población en relación de la otra, los resultados son en expresados en porcentajes (\%) (Matteucci y Colma, 1982).

\section{$D_{2}=(n / N) 100$}

Donde:

$$
\begin{aligned}
& \mathrm{n}=\quad \begin{array}{l}
\text { Número de individuos presente en cada } \\
\text { parcela. }
\end{array} \\
& \mathrm{N}=\quad \text { Suma de todos los árboles presentes. }
\end{aligned}
$$

\section{Frecuencia (F)}

Se utiliza para determinar el nivel de presencia de cada categoría en toda el área de estudio, para estimar la frecuencia se utilizó la fórmula establecida por Matteucci y Colma (1982).

\section{$F=(m / M)-100$}

Donde:

$\mathrm{m}=\quad$ Número de parcelas donde se encuentra la categoría.

$M=\quad$ Número total de unidades muestréales.

\section{Área de copa (Ac)}

El área de copa es una variable importante en estudios de manglares, esta se expresa en $\mathrm{m}^{2}$ y corresponde al área que ocupa la proyección de la copa del árbol sobre el suelo. Esta se calculó con la siguiente fórmula aplicada (Vera, 2020).

Donde:

\section{$\mathrm{Ac}=\pi(\mathrm{Dc})^{2 / 4}$}

$$
\begin{aligned}
& \mathrm{Dc}=\quad \text { Diámetro de copa } \\
& \pi=3,1416
\end{aligned}
$$

\section{Área basal (AB)}

Esta variable expresa el área ocupada por el tronco del árbol, siendo diferente al área de copa, se expresa en $\mathrm{m}^{2}$ por hectárea (ha). En vegetación arborícola se mide a partir de 1,3 m de altura del suelo. Para obtener el valor del área basal se aplicó la fórmula de Carrillo et al. (2008).

\section{$\mathbf{A B}=\pi(\mathrm{DAP} / 2)^{2}$}

Donde:

$$
\begin{aligned}
& \mathrm{DAP}=\begin{array}{l}
\text { Diámetro altura al pecho, que se mide a } 1,3 \\
\mathrm{~m}
\end{array} \\
& \pi=3,1416
\end{aligned}
$$


Rev. Cient. Cien. Nat. Ambien. 14(2):190-200

Diciembre 2020
Moreira - Estado de las poblaciones del mangle blanco Laguncularia racemosa var. glabriflora (Combretaceae) en el Golfo de Guayaquil, Ecuador

\section{Resultados}

Localización de las poblaciones de Laguncularia racemosa var. glabriflora en el Golfo de Guayaquil. En 8 localidades ubicadas en las provincias del Guayas y El Oro se establecieron 10 parcelas rectangulares de $1000 \mathrm{~m}^{2}$, cubriendo una extensión total de 1 hectárea. Se registró un total de 43 individuos con flores y/o frutos de Laguncularia racemosa var. glabriflora en el Golfo de Guayaquil, éstos fueron localizados desde las márgenes de los esteros de la ciudad de Guayaquil en la provincia del Guayas hasta el Puerto Pitayaha en la provincia de El Oro, cerca de los límites políticos del Ecuador en la frontera sur con Perú (Tabla 2).

Patrón de distribución de Laguncularia racemosa var. glabriflora en el Golfo de Guayaquil.

El mangle blanco Laguncularia racemosa var. glabriflora habita predominantemente en manglares ribereños, con una efímera presencia hacia la porción interna en manglares de borde. Se desarrolla preferentemente en manglares de vegetación baja (menores de $5 \mathrm{~m}$ ), y también se puede encontrar en manglares con vegetación de talla media (5 a 20 m) y alta (20 a $40 \mathrm{~m})$ (Tabla 3).

En cuanto al tipo de suelo, esta variedad habita en suelos lodosos limosos de textura floja, pero también puede colonizar en suelos rocosos, alterados por actividad antropogénica, como en las márgenes del estero Palanqueado en la ciudad de Guayaquil, que están constituidas por piedra escollera, cuyos intersticios lodosos son aprovechados en el proceso de establecimiento, germinación y desarrollo hasta llegar al estadío adulto de sus individuos. Además, Laguncularia racemosa var. glabriflora también puede colonizar en suelos artificiales, como el de las islas flotantes del Estero Palanqueado, que están constituidos por plástico poroso reciclado y sin sustrato limoso.

Los individuos censados de L. racemosa var. glabriflora poseen un amplio rango de tolerancia a la salinidad, habitan en sustratos que poseen desde 5 hasta 38 UPS, esto indica que poseen adaptación para habitar en manglares hiposalinos e hipersalinos. Sin embargo, se ha registrado que poseen predominancia por sustratos con salinidad intersticial de 20 a 35 UPS (Fig. 2).

Determinación del estado de las poblaciones de Laguncularia racemosa var. glabriflora en el Golfo de Guayaquil.

Resultados obtenidos en las provincias de Guayas y El Oro, Ecuador.

Se censó un total de 43 individuos distribuidos en el Golfo de Guayaquil, en las provincias Guayas y El Oro. De estos, 34 individuos fueron registrados en ocho parcelas en la provincia del Guayas y 9 en dos parcelas en la provincia de El Oro.

Tabla 2. Localidades por provincia, número de individuos y salinidad intersticial registrada en las parcelas con Laguncularia racemosa var. glabriflora en el Golfo de Guayaquil.

\begin{tabular}{|c|c|c|c|c|c|}
\hline No. & Provincia & Localidad & Posición Geográfica & $\begin{array}{l}\text { No. de individuos } \\
\text { por parcela }\end{array}$ & $\begin{array}{l}\text { Salinidad } \\
\text { intersticial } \\
\text { (UPS) }\end{array}$ \\
\hline 1 & Guayas & $\begin{array}{l}\text { Parque lineal de } \\
\text { Guayaquil }\end{array}$ & $\begin{aligned} 2^{\circ} 11^{\prime} & 3.00 ”, \mathrm{~S} \\
79^{\circ} 54^{\prime} & 0.77^{\prime \prime} \mathrm{W}\end{aligned}$ & 1 & 26 \\
\hline 2 & Guayas & Los helechos & $\begin{array}{c}2^{\circ} 11 ’ 3.52 ”, ~ S \\
79^{\circ} 50^{\prime} 17.32 \text { ' } \mathrm{W}\end{array}$ & 3 & 5 \\
\hline 3 & Guayas & Isla Santay & $\begin{array}{c}2^{\circ} 13^{\prime} 10.35^{\prime \prime} \mathrm{S} \\
79^{\circ} 52^{\prime} 29.58 \text { “' W }\end{array}$ & 3 & 12 \\
\hline 4 & Guayas & Estero Palanqueado & $\begin{array}{c}2^{\circ} 13^{\prime} 27.00 ”, ~ S \\
79^{\circ} 55^{\prime} 23.00 \text { " W }\end{array}$ & 1 & 28 \\
\hline 5 & Guayas & Estero Palanqueado & $\begin{array}{c}2^{\circ} 13^{\prime} 27.00 ”, ~ S \\
79^{\circ} 55^{\prime} 23.00 \text { “' }\end{array}$ & 3 & 28 \\
\hline 6 & Guayas & Estero Palanqueado & $\begin{array}{c}2^{\circ} 13^{\prime} 27.00 ” \mathrm{~S} \\
79^{\circ} 55^{\prime} 23.00 \text { " } \mathrm{W}\end{array}$ & 1 & 28 \\
\hline 7 & Guayas & Puerto de los cangrejeros & $\begin{array}{c}2^{\circ} 25^{\prime} 56.97^{\prime \prime} \mathrm{S} \\
79^{\circ} 39^{\prime} 30.75 \text { ' W }\end{array}$ & 7 & 34 \\
\hline 8 & Guayas & $\begin{array}{l}\text { Sendero de los } \\
\text { cangrejeros }\end{array}$ & $\begin{array}{c}2^{\circ} 26^{\prime} 11.60 ”, ~ S \\
79^{\circ} 39^{\prime} 18.89 \text { ' } \mathrm{W}\end{array}$ & 15 & 25 \\
\hline 9 & El Oro & Isla de Jambelí & $\begin{array}{c}3^{\circ} 14^{\prime} 39.75^{\prime \prime} \mathrm{S} \\
80^{\circ} 2^{\prime} 53.16^{\prime \prime} \mathrm{W}\end{array}$ & 1 & 38 \\
\hline 10 & El Oro & Puerto Pitahaya & $\begin{array}{c}3^{\circ} 25^{\prime} 38.01 \text { ' S } \\
80^{\circ} 4^{\prime} 36.32 \text { " W }\end{array}$ & 8 & 23 \\
\hline
\end{tabular}


Tabla 3. Tipo fisiográfico y alto del bosque de manglar en donde se encuentra Laguncularia racemosa var. glabriflora en las provincias Guayas y El Oro, en el Golfo de Guayaquil.

\begin{tabular}{lllc}
\hline Provincia & Localidad & $\begin{array}{c}\text { tipo fisiográfico } \\
\text { del manglar }\end{array}$ & $\begin{array}{c}\text { alto del bosque } \\
\text { de manglar }\end{array}$ \\
\hline Guayas & Parque Lineal del Estero Salado & Ribereño & Medio \\
& Los helechos & Ribereño & Alto \\
& Isla Santay & Ribereño & Bajo \\
& Estero Palanqueado & Ribereño & Bajo \\
& Estero Palanqueado & Bibereño & Medio \\
El Oro & Puerto de los cangrejeros & Ribereño & Bajo \\
& Sendero de los cangrejeros & Ribereño & Alto \\
\hline
\end{tabular}

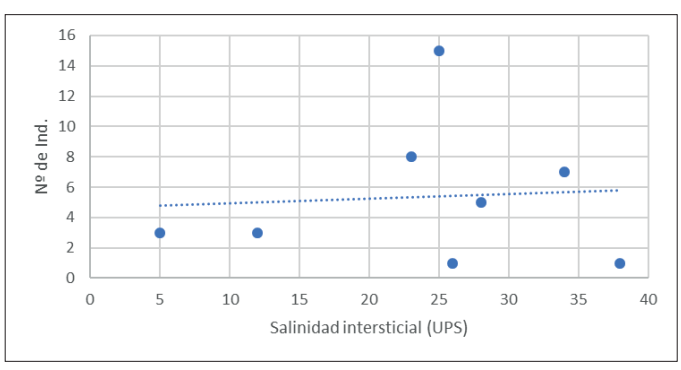

Figura 2. Salinidad intersticial de las localidades censadas con Laguncularia racemosa var. glabriflora en el Golfo de Guayaquil, Ecuador.

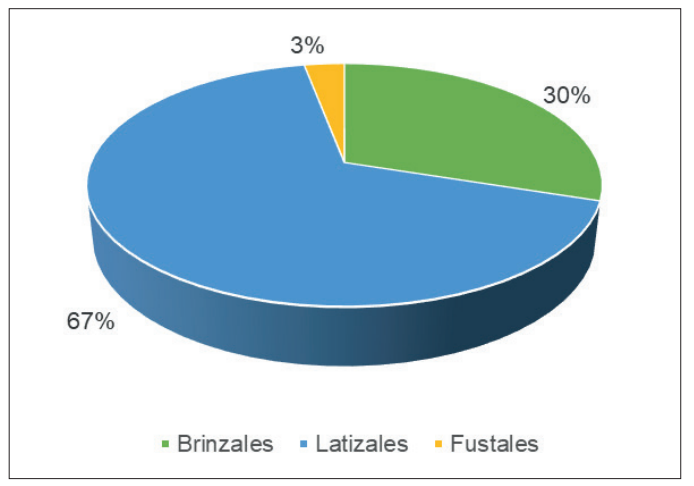

Figura 4. Densidad relativa de las categorías diamétricas de Laguncularia racemosa var. glabriflora en la provincia Guayas, Ecuador.

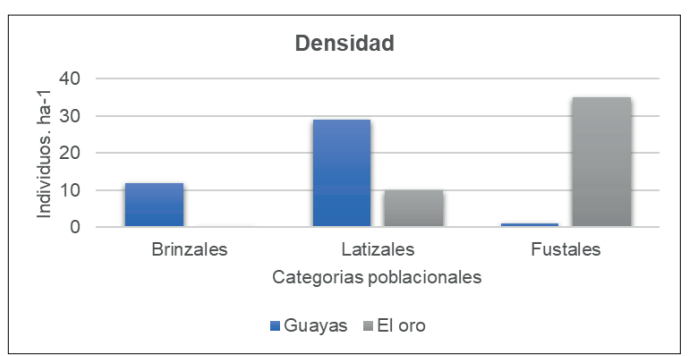

Figura 3. Densidad de las categorías diamétricas de Laguncularia racemosa var. glabriflora en las provincias Guayas y El Oro.

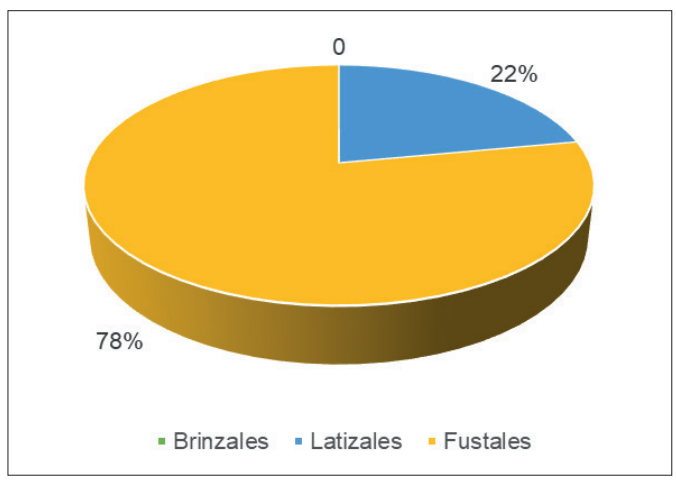

Figura 5. Densidad relativa de las categorías diamétricas de Laguncularia racemosa var. glabriflora en la provincia El Oro, Ecuador.

Tabla 4. Frecuencia de las categorías poblacionales de Laguncularia racemosa var. glabriflora en las provincias Guayas y El Oro, Ecuador.

\begin{tabular}{cccccc}
\hline \multirow{2}{*}{$\begin{array}{c}\text { Categorías } \\
\text { poblacionales }\end{array}$} & $\begin{array}{c}\text { Guayas } \\
\text { N } \mathrm{N}^{\circ} \text { de } \\
\text { parcelas }\end{array}$ & Frecuencia & $\begin{array}{c}\mathrm{N}^{\circ} \text { de } \\
\text { parcelas }\end{array}$ & Frecuencia & Folfo de Guayaquil \\
\hline Brinzales & $6 / 8$ & $75 \%$ & 0 & $0 \%$ & $60 \%$ \\
Latizales & $4 / 8$ & $50 \%$ & $1 / 2$ & $50 \%$ & $50 \%$ \\
Fustales & $1 / 8$ & $13 \%$ & $2 / 2$ & $100 \%$ & $30 \%$ \\
\hline
\end{tabular}


Tabla 5. Área de copa de las categorías poblacionales de Laguncularia racemosa var. glabriflora en las provincias Guayas y El Oro, Ecuador.

\begin{tabular}{|c|c|c|c|}
\hline \multirow{2}{*}{ Categorías poblacionales } & Guayas & El oro & Golfo de Guayaquil \\
\hline & Área de Copa $\left(\mathrm{m}^{2} . \mathrm{ha}^{-1}\right)$ & $\begin{array}{l}\text { Área de Copa } \\
\left(\mathrm{m}^{2} . \mathrm{ha}^{-1}\right)\end{array}$ & Área de Copa $\left(\mathrm{m}^{2} . \mathrm{ha}^{-1}\right)$ \\
\hline Brinzales & 91,22 & 0 & 91,22 \\
\hline Latizales & 118,12 & 12,85 & 130,97 \\
\hline Fustales & 19,64 & 74,98 & 94,62 \\
\hline
\end{tabular}

Tabla 6. Área basal de las categorías poblacionales de Laguncularia racemosa var. glabriflora en las provincias Guayas y El Oro, Ecuador.

\begin{tabular}{cccc}
\hline & Guayas & El oro & $\begin{array}{c}\text { Golfo de } \\
\text { Guayaquil }\end{array}$ \\
\cline { 2 - 4 } Categorías poblacionales & $\begin{array}{c}\text { Área basal } \\
\left(\mathrm{m}^{2} . \mathrm{ha}^{-1}\right)\end{array}$ & $\begin{array}{c}\text { Área basal } \\
\left(\mathrm{m}^{2} . \mathrm{ha}^{-1}\right)\end{array}$ & $\begin{array}{c}\text { Área basal } \\
\left(\mathrm{m}^{2} . \mathrm{ha}^{-1}\right)\end{array}$ \\
\hline Brinzales & 0,12 & 0 & 0,12 \\
Latizales & 0,80 & 0,08 & 0,88 \\
Fustales & 0,12 & 0,75 & 0,87 \\
\hline
\end{tabular}

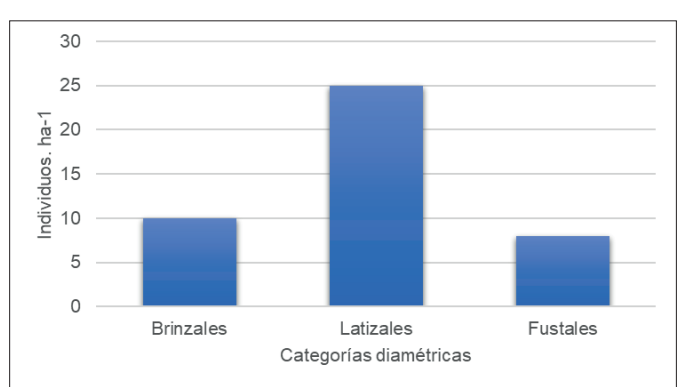

Figura 6. Densidad de las categorías diamétricas de Laguncularia racemosa var. glabriflora en el Golfo de Guayaquil, Ecuador.

Con respecto al DAP, de los 34 individuos censados en la provincia Guayas, 10 pertenecen a la categoría brinzales $(2,5-4,9 \mathrm{~cm}), 23$ a latizales $(5-9,9 \mathrm{~cm})$ y 1 a fustales $(\geq 10 \mathrm{~cm})$. En la provincia El Oro, 2 individuos se encontraban en la categoría latizales y 7 en la categoría fustales y ningún individuo en la categoría brinzales. El DAP máximo registrado para esta variedad es 12,81 cm en Puerto Pitahaya, en la provincia El Oro.

\section{Densidad}

En la provincia Guayas se tiene una densidad de 12 individuos $/ \mathrm{ha}^{-1}$ de la categoría brinzales, 29 individuos $/ \mathrm{ha}^{-1}$ en latizales y 1 individuo $/ \mathrm{ha}^{-1}$ en fustales. En la provincia El Oro, se tiene una densidad de 10 individuos $/ \mathrm{ha}^{-1}$ en latizales y 35 individuos $/ \mathrm{ha}^{-1}$ en fustales (Fig. 3).

\section{Densidad relativa}

La densidad relativa de las categorías diamétricas de Laguncularia racemosa var. glabriflora en las provincias Guayas y El Oro se presenta a continuación (fig. 4,5 , respectivamente).

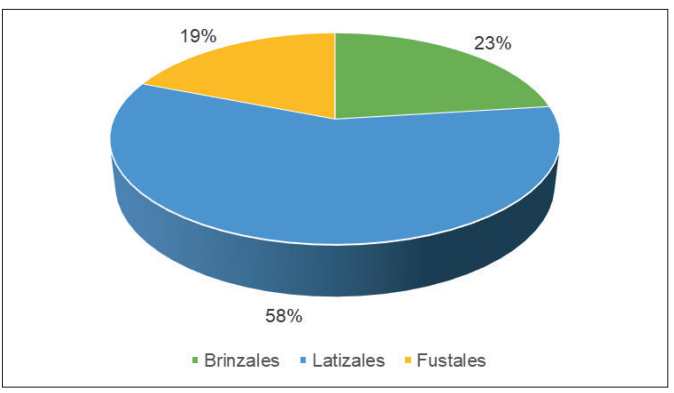

Figura 7. Densidad relativa de las categorías diamétricas de Laguncularia racemosa var. glabriflora en el Golfo de Guayaquil, Ecuador.

\section{Frecuencia $(F)$}

La frecuencia de las categorías poblacionales de Laguncularia racemosa var. glabriflora en las provincias Guayas y El Oro se presenta a continuación (Tabla 4).

\section{Área de Copa}

El área de copa de Laguncularia racemosa var. glabriflora en las provincias Guayas y El Oro se presenta a continuación (Tabla 5).

\section{Área basal}

El área basal de Laguncularia racemosa var. glabriflora en las provincias Guayas y El Oro se presenta a continuación (Tabla 6).

\section{Resultados obtenidos en el Golfo de Guayaquil.}

\section{Densidad}

Los individuos censados de Laguncularia racemosa var. glabriflora corresponden predominantemente a la categoría diamétrica latizales con 25 ind. $/$ ha $^{-1}$, seguido de brinzales con 10 ind. $/$ ha $^{-1}$; estas dos categorías están 
Tabla 7. Alto, DAP, diámetro de copa y estado fenológico de los individuos censados de Laguncularia racemosa var. glabriflora en las localidades visitadas en el Golfo de Guayaquil.

\begin{tabular}{|c|c|c|c|c|c|c|}
\hline $\mathrm{N}^{\circ}$ de Individuos & Localidad & $\operatorname{DAP}(\mathrm{cm})$ & Alto $(\mathrm{m})$ & Diámetro de copa & Flores & Frutos \\
\hline 1 & Parque Lineal & 5 & 2,5 & 3,5 & SI & SI \\
\hline 2 & Los helechos & 12,22 & 2,5 & 5 & SI & SI \\
\hline 3 & Los helechos & 6,63 & 3 & 2,1 & SI & SI \\
\hline 4 & Los helechos & 7,25 & 4 & 3,5 & SI & SI \\
\hline 5 & Isla Santay & 7,28 & 4,5 & 2,6 & SI & SI \\
\hline 6 & Isla Santay & 5,95 & 5 & 2,6 & SI & SI \\
\hline 7 & Isla Santay & 4,71 & 5 & 2,4 & SI & SI \\
\hline 8 & Estero Palanqueado & 4,14 & 3 & 3,7 & SI & SI \\
\hline 9 & Estero Palanqueado & 3,02 & 2,5 & 3,7 & SI & $\mathrm{SI}$ \\
\hline 10 & Estero Palanqueado & 4,03 & 2,5 & 5,5 & SI & SI \\
\hline 11 & Estero Palanqueado & 2,86 & 3 & 3,1 & SI & SI \\
\hline 12 & Estero Palanqueado & 4,62 & 2,5 & 5,1 & SI & SI \\
\hline 13 & Puerto de los cangrejeros & 5,41 & 2,5 & 2,5 & SI & SI \\
\hline 14 & Puerto de los cangrejeros & 7,13 & 4,5 & 2,1 & SI & $\mathrm{SI}$ \\
\hline 15 & Puerto de los cangrejeros & 3,81 & 3 & 2,4 & SI & $\mathrm{SI}$ \\
\hline 16 & Puerto de los cangrejeros & 4,13 & 3 & 1,6 & SI & $\mathrm{SI}$ \\
\hline 17 & Puerto de los cangrejeros & 6,23 & 3 & 2,8 & SI & SI \\
\hline 18 & Puerto de los cangrejeros & 5,46 & 4 & 2,1 & SI & SI \\
\hline 19 & Puerto de los cangrejeros & 5,41 & 3 & 1,6 & SI & $\mathrm{SI}$ \\
\hline 20 & Sendero de los cangrejeros & 5,4 & 6,5 & 2,6 & SI & $\mathrm{SI}$ \\
\hline 21 & Sendero de los cangrejeros & 6,04 & 6 & 2,4 & SI & $\mathrm{SI}$ \\
\hline 22 & Sendero de los cangrejeros & 6,68 & 6,5 & 2,1 & SI & $\mathrm{SI}$ \\
\hline 23 & Sendero de los cangrejeros & 3,19 & 6 & 2,1 & SI & $\mathrm{SI}$ \\
\hline 24 & Sendero de los cangrejeros & 6,62 & 6 & 2,4 & SI & $\mathrm{SI}$ \\
\hline 25 & Sendero de los cangrejeros & 5,28 & 5 & 1,5 & SI & $\mathrm{SI}$ \\
\hline 26 & Sendero de los cangrejeros & 8,1 & 5 & 2,1 & SI & $\mathrm{SI}$ \\
\hline 27 & Sendero de los cangrejeros & 4,8 & 6 & 2,1 & SI & $\mathrm{SI}$ \\
\hline 28 & Sendero de los cangrejeros & 6,04 & 6 & 2,6 & SI & SI \\
\hline 29 & Sendero de los cangrejeros & 6,27 & 6 & 2,6 & SI & $\mathrm{SI}$ \\
\hline 30 & Sendero de los cangrejeros & 8,3 & 6,5 & 2,3 & SI & $\mathrm{SI}$ \\
\hline 31 & Sendero de los cangrejeros & 8,37 & 6 & 2,3 & SI & $\mathrm{SI}$ \\
\hline 32 & Sendero de los cangrejeros & 5,92 & 5,5 & 2,3 & SI & SI \\
\hline 33 & Sendero de los cangrejeros & 8,08 & 5 & 2,5 & SI & SI \\
\hline 34 & Sendero de los cangrejeros & 8,14 & 6,5 & 3,7 & SI & SI \\
\hline 35 & Isla Jambeli & 11,77 & 4,5 & 5,1 & SI & SI \\
\hline 36 & Puerto Pitahaya & 10,15 & 4 & 2,6 & SI & SI \\
\hline 37 & Puerto Pitahaya & 12,54 & 2,5 & 4,5 & SI & SI \\
\hline
\end{tabular}


Continuación Tabla 7. Alto, DAP, diámetro de copa y estado fenológico de los individuos censados de Laguncularia racemosa var. glabriflora en las localidades visitadas en el Golfo de Guayaquil.

\begin{tabular}{ccccccc}
\hline$N^{\circ}$ de Individuos & Localidad & DAP $(\mathrm{cm})$ & Alto $(\mathrm{m})$ & Diámetro de copa & Flores & Frutos \\
\hline 38 & Puerto Pitahaya & 7,7 & 4 & 3,1 & $\mathrm{SI}$ & $\mathrm{SI}$ \\
39 & Puerto Pitahaya & 6,27 & 3 & 2,6 & $\mathrm{SI}$ & $\mathrm{SI}$ \\
40 & Puerto Pitahaya & 10,25 & 3,5 & 3,5 & $\mathrm{SI}$ & $\mathrm{SI}$ \\
41 & Puerto Pitahaya & 12,81 & 4,5 & 3,8 & $\mathrm{SI}$ & $\mathrm{SI}$ \\
42 & Puerto Pitahaya & 12,03 & 3,5 & 2,6 & $\mathrm{SI}$ & $\mathrm{SI}$ \\
43 & Puerto Pitahaya & 11,94 & 3 & 3 & $\mathrm{SI}$ & $\mathrm{SI}$ \\
\hline
\end{tabular}

mayoritariamente presentes en la provincia Guayas, mientras que en la provincia El Oro predomina fustales con una densidad de 8 individuos $/$ ha $^{-1}$ (fig. 6).

\section{Densidad relativa}

La densidad relativa de las categorías diamétricas de Laguncularia racemosa var. glabriflora en el Golfo de Guayaquil se expresan a continuación (fig. 7).

Estado Fenológico de Laguncularia racemosa var. glabriflora

Las poblaciones de Laguncularia racemosa var. glabriflora localizadas e identificadas en la provincia del Guayas y El Oro, presentaron flores y frutos inmaduros en el $100 \%$ de los individuos censados durante diciembre de 2020 y enero de 2021 (Tabla 7).

Además, se registró la presencia de individuos de $L$. racemosa var. racemosa en 8 de las 10 parcelas $(80 \%)$ establecidas en el Golfo de Guayaquil, excluyendo las parcelas del sendero y puerto de los cangrejeros, en donde se registró únicamente $L$. racemosa var. glabriflora.

En cuanto a las parcelas en donde se registró la presencia simpátrica de las dos variedades de $L$. racemosa, se observó en campo que ambas variedades florecen y fructifican simultáneamente, sin presentar individuos con características morfológicas variables o intermedias que pudiera sugerir casos de hibridación entre ambas variedades.

\section{Discusión}

Los resultados del presente estudio confirman la presencia de Laguncularia racemosa var. glabriflora en la provincia del Guayas y El Oro, como lo registró Cornejo (2014), basado en observaciones en campo y colecciones del herbario GUAY. También se determinó una preferencia en el patrón de distribución de este taxón en manglares ribereños, coincidiendo con lo propuesto por Lugo y Snedaker (1974), que determinan que los mangles blancos se desarrollan en manglares que no son influenciados directamente por el oleaje (manglares de borde). Además, se registró una predominancia de individuos por habitar en sustratos lodosos mayormente limosos. En cuanto a la preferencia del grado de desarrollo de la vegetación circundante a la variedad estudiada, se pudo observar que se desarrolla predominantemente en bosques con vegetación baja, es decir que tienen una preferencia por hábitats en donde tiene una mayor influencia de la energía lumínica. Las formaciones vegetales bajas presentaron un menor desarrollo estructural por tratarse de una vegetación más joven, evidenciando el carácter colonizador de L. racemosa var. glabriflora, aún en sustratos predominantemente rocosos y hasta artificiales, como es el caso de las islas flotantes del estero Palanqueado en la ciudad de Guayaquil.

En todas las parcelas se encontró presencia de individuos de $L$. racemosa var. glabriflora con flores y frutos inmaduros, determinando que la floración es favorecida o inducida por los parámetros estacionales al inicio de la estación lluviosa, este evento fenológico es similar a lo previamente registrado para $L$. racemosa var. racemosa en las localidades estudiadas en el presente trabajo, así como en otras localidades como el Estuario de Caeté, Brasil_(Mehlig, 2006). Además, se determina una fuerte simpatría $(80 \%$ de las parcelas censadas) entre las poblaciones de $L$. racemosa var. racemosa y L. racemosa var. glabriflora. En cuanto a este aspecto, llama la atención que pese a presentar una alta similitud de sincronización floral entre ambas variedades, sin embargo, no se encontraron en campo individuos de características morfológicas variables o intermedias que pudiesen indicar una variabilidad morfológica o la presencia de híbridos, demostrando de este modo que $L$. racemosa var. glabriflora es un taxón morfológicamente estable (XC).

\section{Conclusiones}

Los resultados obtenidos en el presente estudio permiten dar las siguientes conclusiones:

Laguncularia racemosa var. glabriflora está presente desde los esteros en la ciudad de Guayaquil en la provincia Guayas, hasta el Puerto de Pitahaya en la provincia El Oro, en Ecuador.

Laguncularia racemosa var. glabriflora se establece predominantemente en manglares ribereños en suelos 
lodosos-limosos de textura floja, pero puede colonizar suelos alterados que mantengan la composición lodosa y aún sustratos artificiales; presenta un amplio rango de tolerancia a la salinidad, desde 5 hasta 38 UPS, siendo más frecuente encontrarla en sustratos entre 20 a 30 UPS.

Esta variedad se desarrolla frecuentemente en lugares en donde la vegetación es baja, inferior a $5 \mathrm{~m}$, teniendo mayor disponibilidad de energía lumínica. Esta característica indica que este es un taxón con una mayor capacidad de captación/secuestro de carbono, por lo que puede ser incluida en proyectos para mitigar el cambio climático.

Las poblaciones de $L$. racemosa var. glabriflora presentes en la provincia Guayas fueron en su mayoría latizales y brinzales, es decir son más jóvenes, con escaso a mediano desarrollo estructural. Mientras que, las poblaciones de la provincia El Oro tienen un mayor desarrollo estructural al dominar en la categoría fustales, lo que indica que se trata de individuos de mayor edad y en mejor estado de conservación.

La presencia de flores y frutos inmaduros de $L$. racemosa var. glabriflora durante diciembre 2020 y enero 2021, sugieren una relación con el inicio de la estación lluviosa y determinan que la fenología de este taxón está condicionada en función de los parámetros físicos estacionales.

La fuerte simpatría ( $80 \%$ de las parcelas censadas) y sincronización floral entre Laguncularia racemosa var. racemosa y la variedad glabriflora, sin producción de individuos con características morfológicas variables o intermedias como ha sido observado en campo y en las colecciones de herbario, permiten concluir que no hay indicios de variabilidad morfológica ni de hibridación entre las dos variedades de mangle blanco. Esto demuestra que $L$. racemosa var. glabriflora es un taxón morfológicamente estable, por lo que debe revisarse su estatus de variedad.

\section{Recomendaciones}

- Los resultados y conclusiones obtenidas permiten proveer las siguientes recomendaciones:

- Realizar estudios para determinar el comportamiento fenológico anual y a largo plazo de Laguncularia racemosa var. glabriflora.

- Realizar estudios moleculares que permitan obtener información relevante sobre el linaje genético y que sirvan de soporte para la revisión del estatus taxonómico y nomenclatural de Laguncularia racemosa var. glabriflora.

- Realizar estudios poblacionales comparativos entre las dos variedades de $L$. racemosa, para determinar la abundancia relativa de cada variedad en los bosques de manglar.
- Evaluar el potencial de captación de carbono de Laguncularia racemosa var. glabriflora y la factibilidad de ser incluida en los planes y estrategias para mitigar el cambio climático.

\section{Bibliografía}

Benavides A., Del Pezo, R., Pernia, B., Mero, M., Cornejo, X., Magallanes, y I., Romero, B. 2018. Effect of cadmium on the germination and growth of Laguncularia racemosa var. glabriflora (Plantae-Combretaceae)/Efecto del cadmio sobre la germinación y crecimiento de Laguncularia racemosa var. glabriflora (Plantae-Combretaceae). Ecovida 8(1): 64-83.

Benítez-Pardo, Daniel, Benítez-Hernández, Asahel, Flores-de-Santiago, Francisco, y Amador-Cruz, F. 2018. Contenido nutrimental en hojas de Laguncularia racemosa (Combretaceae), relacionado con su fenología en una laguna tropical del Golfo de California, México. Acta Botánica Mexicana 124: 227-234.

Bravo, M. 2010. Interpretación del estudio multitemporal (CLIRSEN 1969-2006) de las coberturas de manglar, camaroneras y áreas salinas en la franja costera del Ecuador continental. Tesis de grado para la obtención de título de Magister en ciencias con énfasis en Manejo Sustentable de Recursos Bioacuáticos y Medio Ambiente, Universidad de Guayaquil.

Carrillo, A., Elizalde-Rendón, E., Torrescano, N., y Flores, G. 2008. Adaptación ante disturbios naturales, manglar de Puerto Morelos, Quintana Roo, México. Foresta Veracruzana 10(1): 31-38.

Cintrón, G.M. y Schaeffer, Y. 1992. Ecology and management of New World mangroves. En: U. Seeliger, ed. Coastal plant communities in Latin America, pp. 233-258. Academic Press, San Diego.

Cornejo, X. 2014. Plants of the South American Pacific Mangrove Swamps (Colombia, Ecuador, Peru). Ed. EduQuil. Universidad de Guayaquil. Ecuador. 310 p.

Cornejo, X. 2015. Las especies emblemática de flora y fauna de la ciudad de Guayaquil y de las provincias del Guayas, Ecuador. Revista Científica de Ciencias Naturales y Ambientales 9: 56-71.

Cumaná, L., Sanabria, M. E., Leopardi, C., y Guevara de Franco, Y. 2010. Plantas vasculares de los manglares del estado Sucre, Venezuela. Acta Botánica Venezuelica 33: 273-298.

DINAF-CLIRSEN. 1991. Inventario de manglares de Ecuador continental. Quito, Ecuador.

Erazo, A. 2014. Uso estratégico del mangle para el desarrollo turístico en el cantón San Lorenzo, provincia de Esmeraldas. Tesis de grado previa a la obtención del título de licenciada en Turismo Ecológico, Universidad Central del Ecuador, Quito.

Garrido, S. 1993. Interpretación de análisis de suelos. Hojas de Divulgación Núm. 5. Pág. 19.

Idrogo, I. 2016. Estructura de las especies de mangle en el Santuario Nacional de los manglares de Tumbes. Tesis para optar el título de Biólogo, Universidad de Nacional de Piura. 
INOCAR. 2021. Tabla de mareas puertos del Ecuador. Disponible en: https://www.inocar.mil.ec/web/index. $\mathrm{php} /$ productos/tabla-mareas

Lugo, A. E. y S. C. Snedaker. 1974. The Ecology of mangroves. Annual Review of Ecology and Systematics 5: 39-64.

Madsen, J.E., R. Mix \& H. Baslev. 2001. Flora of Puna Island: Plant resources on a Neotropical island. Aarhus University Press. 289 pp.

Matteucci, S. y A. Colma. 1982. Metodología para el estudio de la vegetación. Secretaría General de la Organización de los Estados Americanos. Programa Regional de Desarrollo Científico y Tecnológico. Washington, D.C. U.S.A.

Mehlig, U. 2006. Phenology of the red mangrove, Rhizophora mangle L., in the Caeté Estuary, Pará, equatorial Brazil. Aquatic Botany 84: 158-164.

Murrieta, M. 2018. Evaluación de la velocidad de descomposición de hojarasca de Laguncularia racemosa (L.) C.F. Gaerth., Avicennia germinans (L.) y Rhizophora mangle (L.) en el refugio de vida silvestre manglar del estuario del río Esmeraldas. Tesis previa a la obtención de título de Ingeniero de Gestión Ambiental, Pontificia Universidad Católica del Ecuador, Esmeraldas.

Presl, K.B. 1825-1835. Reliquiae Haenkeanae, seu descriptiones et icones plantarum, quas in America Meridionali et Boreali, in insulis Philippines et Marianis collegit Thaddaeus Haenke (reprint 1973). Amsterdam.

Rendón, M. 2020. Detección de cambios de la cobertura vegetal, mediante teledetección en el sitio RAMSAR manglares del estuario interior del Golfo de Guayaquil "Don Goyo". Trabajo de titulación para la obtención del título de Ingeniera Ambiental, Universidad de Guayaquil.

Stace, C. A. 2007. Combretaceae. En: G. Harling \& C. Persson, Flora of Ecuador, University of Göteborg, 81:1-63.

Vera, J. 2020. Cuantificación de carbono almacenado en el manglar del acuerdo de uso sustentable y custodia en Puerto Libertad, Provincia del Guayas, Ecuador. Trabajo de titulación previo a obtener el grado académico de Ingeniero Ambiental, Universidad de Guayaquil. 